\title{
Anal Squamous Cell Carcinoma Presenting as Perineal Sepsis: A Case Report and Literature Review
}

\author{
Ricardo Balanzá ${ }^{1}$, Pedro Cuevas², Ximena Zozaya ${ }^{1}$, Manuel Alejandro Hay ${ }^{1}$ and Lourdes Molinar ${ }^{3}$ \\ Department of General Surgery, Hospital Angeles Lomas, México \\ Department of Colorectal Surgery, Hospital Angeles Lomas, México \\ Department of Pathology, Hospital Angeles Lomas, México
}

Submission: July 14, 2017; Published: July 28, 2017

*Corresponding author: Ricardo Balanzá, Department of General Surgery, Hospital Angeles Lomas, México, Tel: 04455446045 164; Email: balanza.ricardo@gmail.com

\begin{abstract}
Background: Anal canal cancer is an uncommon cancer worldwide; nevertheless its incidence has risen in recent years. Squamous carcinoma is the most common histological type of anal canal cancer. The most important risk factor for squamous anal carcinoma is infection by oncogenic types of human papillomavirus. The clinical presentation for this malignancy usually includes pain, bleeding, swelling, pruritus, and discharge. The treatment goals for anal cancer include local control, ideally with sphincter preservation. The management of this malignancy has evolved from surgery to curative chemoradiation, with radical surgery as a salvage treatment.

Case report: A 53-year-old female patient presented to the emergency department with severe pain localized to the perineal area, associated with transanal bleeding, purulent drainage, fever, and the sensation of a bulge near the anus. Proctological exploration showed a left lateral swelling with erythema and a secondary opening with haemopurulent drainage, digital rectal examination revealed a left lateral mass with minimal fluctuation. A rectal exam under anesthesia was performed identifying an ulcerated mass with an associated abscess and fistulous tract. Biopsies of the indurated ulcer with rolled up borders were obtained. The pathology report revealed an invasive squamous carcinoma. We performed a CT-scan to classify the tumor as T3NOMO corresponding to NCCN clinical stage II. Serology for HIV was negative. Biopsy samples were negative for HPV infection. The patient underwent chemoradiation with Mitomycin C and Capecitabine with complete clinical response.
\end{abstract}

Keywords: Anal cancer; Anal squamous cell carcinoma; Perineal sepsis; Human papillomavirus; Chemoradiation

\section{Introduction}

Anal cancer represents approximately $1 \%$ of all gastrointestinal cancers [1]. The incidence of anal cancer around the world has risen over the last 30 years [1,2]. Anal squamous cell carcinoma (ASCC) represents between 80 and $85 \%$ of all anal canal cancers [1,2]. The oncogenic types of human papillomavirus (HPV) are etiologically linked to ASCC [1]. Risk factors for this malignancy are HPV infection, human immunodeficiency virus (HIV) infection, immune suppression in transplant recipients, smoking, receptive anal intercourse, lifetime number of sexual partners, and previous in situ or invasive cervical, vulvar or vaginal cancer [3]. Anal cancer screening is being stablished for high risk populations, such as HIV-positive individuals, in the form of anal Papanicolaou and high-resolution anoscopy [4]. ASCC can present with any combination of a mass, non-healing ulcer, bleeding, pain, pruritus, discharge, fistula, or fecal incontinence [5]. Radiation with concurrent chemotherapy is considered as the standard of care for ASCC [6]. The combination of 5-Fluorouracil (5-FU) and other cytotoxic agents as Mitomycin C (MMC) is able to achieve complete tumor regression in up to $90 \%$ of patients [5].

\section{Case Report}

A 53-year-old female patient presented to the emergency room with the chief complaint of perineal pain for the last 3 days. The pain was constant, severe, localized to the perineal area, associated to transanal bleeding, purulent drainage, fever, and the sensation of a bulge near the anus. The temperature was $38.4^{\circ} \mathrm{C}$, heart rate was $106 / \mathrm{min}$, respiratory rate was $24 / \mathrm{min}$, and blood pressure was $90 / 50 \mathrm{~mm} \mathrm{Hg}$. On physical examination the patient was dehydrated, the abdomen was flat, with normal bowel sounds, perianal inspection showed a left lateral swelling with erythema, a linear ulcer with rolled up edges suggestive of malignant disease, and a fistula opening with haemopurulent drainage, digital rectal examination revealed a left lateral mass with minimal fluctuation around approximately $40 \%$ of the anal canal. Initial blood count and blood chemistry tests were entirely normal except for an elevated leukocyte count at $18,000 / \mathrm{mm}^{3}$ with shift to the left. Past medical and surgical history were not significant. We decided to proceed to the operating room for drainage and biopsy of the mass with the suspected diagnosis of a tumor associated fistula. 
A rectal exam under anesthesia was performed identifying an ulcerated mass of approximately $6 \times 4 \times 3 \mathrm{~cm}$ in the left lateral position of the anal canal with an associated abscess and fistulous tract. The tumor was classified as T3NXMX at the time of surgery. Biopsies were obtained by quadrants to map the extent of the lesion, including the transition to normal tissue macroscopically, the abscess was drained and a fistulectomy was performed along with resection of the engorged hemorrhoidal tissue around the ulcer (Figure 1). A rigid sigmoidoscopy showed the absence of other lesions up to $25 \mathrm{~cm}$. The pathology report revealed fragments of the anal canal with focally keratinizing moderately differentiated invasive squamous carcinoma and multifocal squamous carcinoma in situ (Figure 2 \& 3). Human papillomavirus was not detected in the biopsies. Human immunodeficiency virus serology was negative for types 1 and 2 .

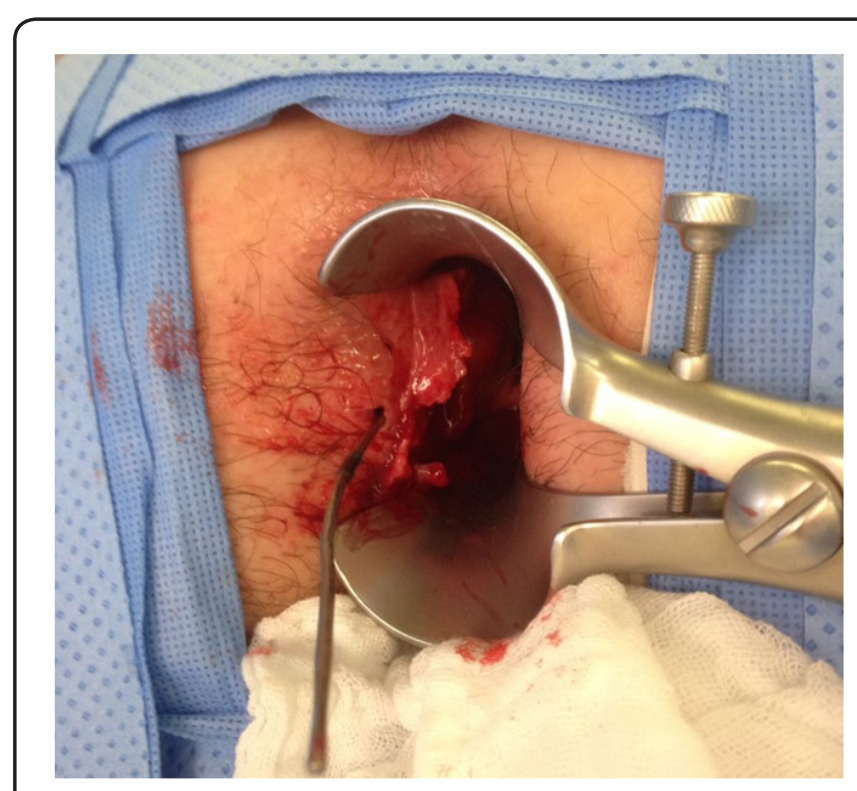

Figure 1: Abscess drainage and fistulectomy.

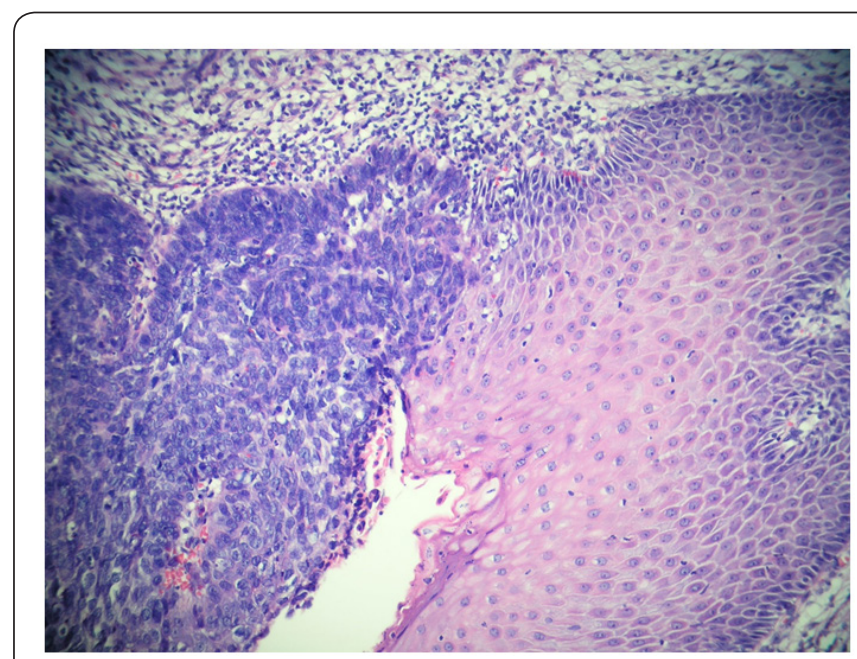

Figure 2: Hematoxylin and eosin staining showing a squamous anal carcinoma in situ.

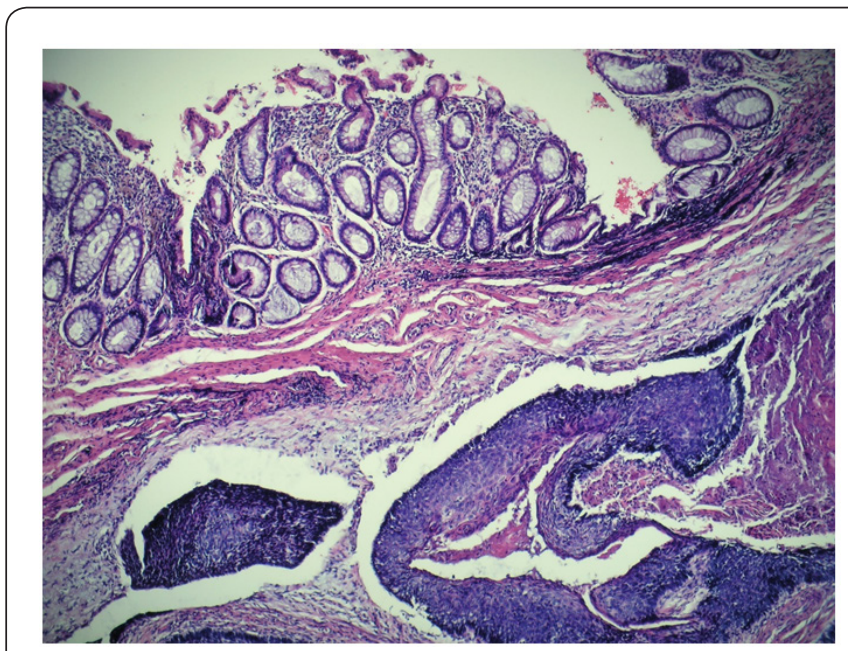

Figure 3: Hematoxylin and eosin staining showing a moderately differentiated invasive squamous anal carcinoma.

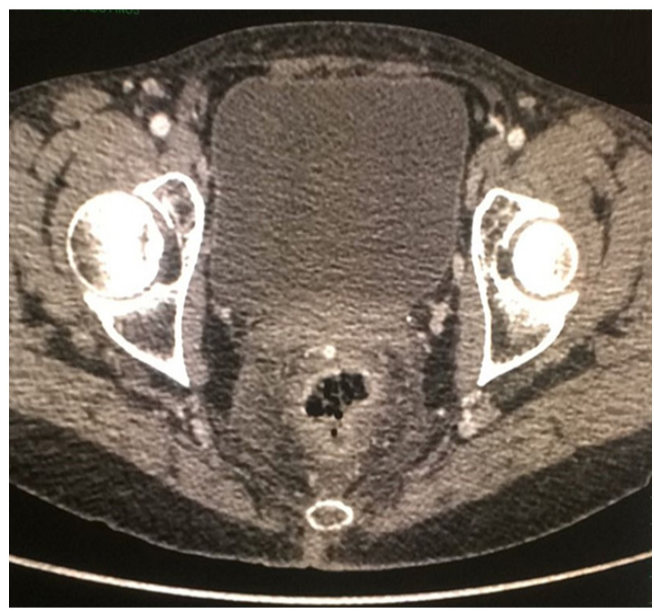

Figure 4: CT scan showing a tumor $>5 \mathrm{~cm}$ in the anal canal with no regional lymph node or distant metastasis (T3NOMO).

A computed tomography (CT scan) of the abdomen and pelvis was used to stage the tumor as T3N0M0 with clinical stage II (Figure 4). A Pap smear and colposcopy were performed with negative results. The patient initiated modified Nigro protocol with Mitomycin C and Capecitabine along with radiotherapy. The patient had a positron emission tomography (PET/CT) 4 months after completing chemoradiation with no evidence of persistent disease. She will have a follow-up with proctologic examination every 3 months and a PET/CT every 6 months.

\section{Discussion}

Anal cancer is a relatively uncommon cancer and accounts for $0.43 \%$ of all malignancies [4]. The incidence of this cancer has risen in the United States from 0.8 to 1.7 cases per 100,000 persons per year from 1975 to 2011 [4]. The incidence of anal cancer in England in 2010 was 1.2 per 100,000 persons per year in men and 1.6 per 100,000 persons per year in women [2]. The 
mean age for diagnosis in anal cancer is 60 years and only $1.1 \%$ of the cases are diagnosed in people younger than 35-years-old [7]. Anal cancer is a term that includes both anal canal and anal margin tumors [2]. The anal canal is defined as the terminal part of the large intestine extending from the anorectal junction in the upper part of the pelvic floor to the anal verge, which is the hairbearing skin around the anus [4]. The anal margin is defined as the area extending from the anal verge to $5 \mathrm{~cm}$ circumferentially outward on the perineal skin [2].

The predominant histological type of anal canal cancer is ASCC, which constitutes between 80 and $85 \%$ of all anal canal carcinomas [1,2]. Other histological types of anal canal cancer include adenocarcinomas, which account for between 5 and $18 \%$ of anal cancers, mucinous adenocarcinomas, small cell carcinomas, undifferentiated carcinomas, carcinoid tumors, non epithelial tumors, and melanomas [2]. Factors that increase the risk of presenting ASCC include HPV infection, anal intercourse, high lifetime number of sexual partners, HIV infection, immune suppression in transplant recipients, use of immune suppressants such as long-term corticosteroids, a history of other HPV-related cancers, autoimmune disorders, social deprivation, and cigarette smoking [5]. The anal cancer incidence in 2012 for HIV-infected men-who-have-sex-with-men (MSM) in North America was 131 cases per 100,000 persons [8]. Anoreceptive intercourse among heterosexual women is a poorly quantified factor believed to contribute to the increase in frequency of ASCC [7].

ASCC has etiological similarities with some genital and oropharyngeal malignancies, sharing the association between cancer with HIV and HPV [9]. ASCC is associated with the oncogenic subtypes of HPV in up to $90 \%$ of the cases $[2,10]$. The prevalence of HPV infection in the United States ranges from between $10 \%$ to $55 \%$ in the general population and approaches $90 \%$ in HIV-infected individuals [1]. HPV infection is characterized by the over expression of viral oncogenes E6 and E7 [11]. E6/E7 oncoproteins block the tumor suppressors p53 and Rb leading to cell-cycle disruption and accumulation of chromosomal instability [11]. Increased E7 expression is also responsible for epigenetic remodeling of the p16INK4a gene locus, whose over expression results in senescence and cell-cycle arrest [11]. Anal high-grade squamous intraepithelial lesions (HSIL), caused by HPV infection, are postulated to be the precursors of ASCC [8]. HPV 16 is the most common subtype of HPV linked to anal carcinogenesis and is present in $75 \%$ of invasive ASCC [2].

Cervical cancer and ASCC are biologically similar and caused by persistent infection with high-risk types of HPV [12]. Screening strategies used in cervical cancer have been applied to anal cancer in the form of anal cytology (anal Pap smear) and high-resolution anoscopy, which is similar to colposcopy [12]. Anal cytology is performed without direct visualization of the anal canal with a moistened Dacron swab, a cervical brush or a flocked nylon swab, the implement is inserted $4 \mathrm{~cm}$ into the anal canal and rotated around its full circumference [7]. Anal cytology is reported using the terminology and definitions of the Bethesda System [7]. The sensitivity of a single anal cytology test for detection of histological HSIL ranges from 55 to $93 \%$ and the specificity from 32 to $81 \%$ [7]. Anal cytology should be done every 12 months in HIV-positive men and women over 25- yearsold, HIV-negative MSM over 40-years-old, women with highgrade cervical or vulvar lesions or cancer over 40-years-old, men and women with perianal condyloma or HSIL over 25-years-old, and solid organ transplantation recipients or patients with other forms of immune suppression over 25-years-old [13]. High resolution anoscopy with biopsy is indicated in cases where anal cytology results are abnormal or anal cancer symptoms are present and has a sensitivity and specificity of $100 \%$ and $71 \%$ respectively [7].

Patients with ASCC can present with a variety of perianal symptoms, but usually present with a combination of pain, bleeding, swelling, pruritus, and discharge [2]. Bleeding is a common symptom for ASCC and is often attributed to hemorrhoids, delaying the diagnosis [5]. Symptoms that suggest advanced disease include fecal incontinence, pelvic pain, and the presence of a rectovaginal fistula [2]. Inguinal metastasis may be present in up to $29 \%$ of patients at presentation [2]. The diagnosis of ASCC is based on biopsy results [5]. In order to adequately stage ASCC after histological confirmation a CT scan or magnetic resonance imaging (MRI) of the chest, abdomen, and pelvis is required [2]. Endo-anal ultrasound (EUS) is useful for $\mathrm{T}$ stage but inadequate for $\mathrm{N}$ and $\mathrm{M}$ staging [5]. The United States National Comprehensive Cancer Network guidelines recommend PET/CT to be considered in the staging of patients with node-positive disease or T3-4 primary tumors [14].

Patients with diagnosis of HSIL can be treated with infrared coagulation or electrocautery ablation reaching cure rates for individual lesions of $67 \%$ in HIV-positive patients and $80 \%$ in HIV-negative patients [15]. HPV 16 positivity in ASCC is associated with a better outcome, being an independent prognostic factor for overall survival and disease-specific survival [3]. Historically, treatment for ASCC was abdominoperineal resection with formation of a permanent stoma [16]. Eradication of the primary tumor with preservation of the structure and function of the anal sphincters are the modern treatment goals of ASCC [2]. The actual standard therapy for ASCC is definitive chemoradiotherapy delivering 50-60 Gy with concurrent 5-FU/ MMC (17). Usual treatment for ASCC produce a 35\% rate of grade 3 to 4 gastrointestinal toxicity and a $48 \%$ rate of skin toxicity [17]. Patients treated with combined chemoradiotherapy can experience ongoing distressing symptoms such as diarrhea, fecal leakage, incontinence, and poor sexual function for many years after treatment $[16,18]$.

Capecitabine is an oral, tumor-activated fluoropyrimedine carbamate, preferentially converted to 5-FU at the tumor site, which avoids the need for hospital admission and a central 
line for the administration of 5-FU [19]. Patients treated with Capecitabine present less mucositis, nausea, and neutropenia than those treated with 5-FU [20]. Cost comparison of Capecitabine versus 5-FU shows that the higher drug cost of Capecitabine is offset by the costs associated with treating toxicity and administrating 5-FU (20). Intensity-modulated radiation therapy can decrease the dose administered to normal structures, while maintaining adequate ASCC coverage [18]. The 5-year survival rate for ASCC is 79\%, 5-year colostomy free survival rate ranges from 65 to $82 \%$, 5-year disease-free survival rate is $58 \%$, and 5-year progression-free survival rate is $78 \%$ [2]. Salvage surgery can achieve local pelvic control in approximately $60 \%$ of cases with a 5 -year survival rate of 30 to $60 \%$ [5]. There are no definitive guidelines on anal cancer follow-up after chemoradiotherapy [2].

\section{Conclusion}

Our patient presented to the emergency department with a combination of perianal symptoms, including the presence of a mass, fistula, pain, discharge, and bleeding, which are all suggestive of anal cancer. The diagnosis of ASCC was made by biopsy-proven histology taken during a rectal exam under anesthesia. In order to adequately stage the tumor we performed a CT scan of the abdomen and pelvis, along with a full vaginal exploration. The ASCC was classified as T3N0M0 with clinical stage II. HIV and HPV infection were ruled out because of the association of these viruses with ASCC. Our patient was treated with chemoradiotherapy using Capecitabine instead of 5-FU to avoid the need for hospital admission and a central line. Although there is no definitive guideline on anal cancer followup, we performed a PET/CT 4 months after treatment ended and will carry out clinical examination every 3 months with repeat imaging every 6 months with a low threshold for biopsy. There is no evidence of persistent or recurrent disease 7 months after treatment.

\section{TTM-097-001 and CSMU-TTM-098-002.}

\section{References}

1. Nelson RA, Levine AM, Bernstein L, Smith DD, Lai LL (2013) Changing Patterns of Anal Canal Carcinoma in the United States. J Clin Oncol 31(12): 1569-1575

2. Bown E, Shah V, Sridhar T, Boyle K, Hemingway D, et al. (2014) Cancers of the anal canal: diagnosis, treatment and future strategies. Future Oncol 10(8): 1427-1441.

3. Hansen ES, Linnemann D, Ruminski WS, Hogdall E, Flemming PG, et al. (2014) Human Papillomavirus Genotyping and p16 Expression As Prognostic Factors for Patients With American Joint Committee on Cancer Stages I to III Carcinoma of the Anal Canal. J Clin Oncol 32(17): 1812-1817.

4. Shridhar R, Shibata D, Chan E, Thomas CR (2015) Anal Cancer: Current Standards in Care and Recent Changes in Practice. CA Cancer J Clin 65(2): 139-162
5. Jones RG, Nilsson PJ, Aschele C, Goh V, Peiffert D, et al. (2014) Anal cancer: ESMO-ESSO-ESTRO clinical practice guidelines for diagnosis, treatment and follow-up. Radiother Oncol 111(3): 330-339.

6. Amini A, Jones BL, Ghosh D, Schefter TE, Goodman KA (2016) Impact of Facility Volume on Outcomes in Patients With Squamous Cell Carcinoma of the Anal Canal: Analysis of the National Cancer Data Base. Cancer 123(2): 228-236.

7. Moscicki AB, Darragh TM, Berry-Lawhorn M, Roberts JM, Khan MJ, et al. (2015) Screening for Anal Cancer in Women. J Low Genit Tract Dis 19(3): 27-42.

8. Berry JM, Jay N, Cranston RD, Darragh TM, Holly EA, et al. (2014) Progression of anal high-grade squamous intraepithelial lesions to invasive anal cancer among HIV-infected men who have sex with men. Int J Cancer 134(5): 1147-1155.

9. Koerber SA, Schoneweg C, Slynko A, Drug D, Haefner MF, et al. (2014) Influence of human papillomavirus and $\mathrm{p} 16^{\text {(INK4a) }}$ on treatment outcome of patients with anal cancer. Radiother Oncol 113(3): 331-336.

10. Jones CM, Goh V, Montefiore DS, Gilbert DC (2017) Biomarkers in anal cancer: from biological understandinig to stratified treatment. $\mathrm{Br} \mathrm{J}$ Cancer 116(2): 156-162.

11. Mai S, Welzel G, Ottstadt M, Lohr F, Severa S, et al. (2015) Prognostic Relevance of HPV Infection and p16 Overexpression in Squamous Cell Anal Cancer. Int J Radiat Oncol Biol Phys 93(4): 819-827.

12. Patel J, Salit IE, Berry MJ, de Pokomandy A, Nathan M, et al. (2014) Environmental scan of anal cancer screening practices: worldwide survey results. Cancer Med 3(4): 1052-1061.

13. Palefsky JM (2015) Screening to Prevent Anal Cancer: Current Thinking and Future Directions. Cancer Cytopathol 123(9): 509-510.

14. Jones M, Hruby G, Solomon M, Rutherford N, Martin J (2015) The Role of FDG-PET in the Initial Staging and Response Assessment of Anal Cancer: A Systematic Review and Meta-analysis. Ann Surg Oncol 22(11): 3574-3581.

15. Goldstone SE, Johnstone AA, Moshier EL (2014) Long-term Outcome of Ablation of Anal High-grade Squamous Intraepithelial Lesions: Recurrence and Incidence of Cancer. Dis Colon Rectum 57(3): 316-323.

16. Knowles G, Haigh R, McLean C, Phillips H (2015) Late effects and quality of life after chemo-radiation for the treatment of anal cancer. Eur J Oncol Nurs 19(5): 479-485.

17. Mitchell MP, Abboud M, Eng C, Beddar AS, Krishnan S, et al. (2014) Intensity-modulated Radiation Therapy With Concurrent Chemotherapy for Anal Cancer. Am J Clin Oncol 37(5): 461-466.

18. Benson AB, Arnoletti JP, Saab TB, Chan E, Chen YJ, et al. (2012) Anal Carcinoma, Version 2.2012: featured updates to the NCCN guidelines. J Natl Compr Canc Netw 10(4): 449-454.

19. Jones RG, Meadows H, Wan S, Gollins S, Leslie M, et al. (2008) Extra-A Multicenter Phase II Study of Chemoradiation Using a 5 Day Per Week Oral Regimen of Capecitabine and Intravenous Mitomycin C in Anal Cancer. Int J Radiat Oncol Biol Phys 72(1): 119-126. 
20. D’Alpino R, Wan DD, Schellenberg D, Lim HJ (2016) A comparison between 5-fluorouracil/mitomycin and capecitabine/mitomycin in

This work is licensed under Creative

Commons Attribution 4.0 Licens

DOI:10.19080/JOJCS.2017.03.555621 combination with radiation for anal cancer. J Gastrointest Oncol 7(4): 665-672.

\section{Your next submission with Juniper Publishers will reach you the below assets}

- Quality Editorial service

- Swift Peer Review

- Reprints availability

- E-prints Service

- Manuscript Podcast for convenient understanding

- Global attainment for your research

- Manuscript accessibility in different formats ( Pdf, E-pub, Full Text, Audio)

- Unceasing customer service

Track the below URL for one-step submission https://juniperpublishers.com/online-submission.php 